\title{
Linx
}

Revue des linguistes de l'université Paris X Nanterre

56 | 2007

Linguistique des genres

\section{Le genre en linguistique de discours comparative. Stabilités et instabilités séquentielles et énonciatives}

Patricia von Münchow

\section{(2) OpenEdition \\ Journals}

Édition électronique

URL : http://journals.openedition.org/linx/370

DOI : $10.4000 /$ linx.370

ISSN : 2118-9692

Éditeur

Presses universitaires de Paris Nanterre

Édition imprimée

Date de publication : 1 juin 2007

Pagination : 109-125

ISSN : 0246-8743

\section{Référence électronique}

Patricia von Münchow, «Le genre en linguistique de discours comparative. Stabilités et instabilités séquentielles et énonciatives », Linx [En ligne], 56 | 2007, mis en ligne le 21 février 2011, consulté le 21 avril 2019. URL : http://journals.openedition.org/linx/370 ; DOI : 10.4000/linx.370 


\title{
Le genre en linguistique de discours comparative. Stabilités et instabilités séquentielles et énonciatives
}

\author{
Patricia von Münchow \\ Université Paris Descartes - DYNALANG
}

\begin{abstract}
Dans cet article, je m'efforcerai de montrer le rôle primordial, sur plusieurs plans, que joue le concept de "genre» dans une approche relevant des sciences du langage que j'appelle « linguistique de discours comparative », de même que le rôle que joue la linguistique de discours comparative dans la description de différents genres ainsi que pour une meilleure compréhension de leur fonctionnement. Après avoir brièvement exposé le cadre théorico-méthodologique de cette approche, je ferai état des résultats de l'analyse comparative de cinq genres de l'écrit du point de vue des types de séquences textuelles et du discours rapporté.
\end{abstract}

\section{Quelques éléments théorico-méthodologiques}

\subsection{Le genre en linguistique de discours comparative}

En linguistique de discours comparative telle que je la conçois, il s'agit de comparer différentes cultures discursives par l'intermédiaire des productions verbales qui en relèvent. Pour y parvenir, je mets en rapport les manifestations d'un même genre discursif dans au moins deux communautés ethnolinguistiques différentes, genre 
dont il s'agit alors de décrire les invariabilités (ou «stabilités») et les variabilités (ou « instabilités»), aux niveaux compositionnel, énonciatif et sémantique. Les caractéristiques relevées sont ensuite reliées, dans un mouvement interprétatif, aux fonctions du genre et aux représentations (mentales) qui circulent parmi les locuteurs et les récepteurs. À leur tour et par l'interprétation de l'analyste, là encore, les fonctions et les représentations sont mises en relation avec des « causalités » institutionnelles, culturelles, etc.

L'un des rôles du genre en linguistique de discours comparative est celui de tertium comparationis. Mais le genre ne rend pas seulement possible la comparaison, il est aussi l'entité qui constitue la fin - provisoire - de la description/interprétation. La raison pour laquelle la comparaison effective se situe au niveau des genres discursifs et non pas - immédiatement - à celui des communautés ethnolinguistiques, autrement dit la raison pour laquelle ce que j'ai appelé le "niveau de représentativité »" de l'analyse en linguistique de discours comparative est le genre, est que, comme le dit Bakhtine (1984, p. 284), « [1] vouloir-dire du locuteur se réalise avant tout dans le choix d'un genre du discours. /.../ Après quoi, le dessein discursif du locuteur, sans que celui-ci se départisse de son individualité et de sa subjectivité, s'adapte et s'ajuste au genre choisi, se compose et se développe dans la forme du genre donné. » Pour Rastier (1998, p. 101), « les structures textuelles dépendent moins du système fonctionnel de la langue que d'autres systèmes de normes (notamment les genres et les styles). » Ainsi le genre est-il une catégorie contraignant fortement la matérialité discursive, ce qui incite à rapporter les résultats de l'analyse au niveau de représentativité " genre », c'est-à-dire à les considérer comme représentatifs de ce niveau et non pas d'un niveau «supérieur » comme le «champ générique », le « discours » $»^{2}$, voire une langue-culture dans son ensemble. Toute généralisation de la portée des résultats d'analyse vers un niveau de représentativité «supérieur » est a priori abusive et ne pourra se fonder à terme que sur l'analyse d'un nombre important de genres.

Aussi bien le statut de tertium comparationis que celui de niveau de représentativité (provisoire) posent une série de problèmes théorico-méthodologiques, que je me suis efforcée de résoudre, pour rendre la catégorie "genre » opératoire, en m’inspirant de différents travaux provenant de cadres théoriques divers. En amont de l'analyse, les différents genres - qui constituent des catégories prototypiques - sont difficiles à délimiter les uns par rapport aux autres, ce qui pose un problème d'établissement du corpus, d'une part, et l'aspect translangagier du genre ne peut pas être considéré comme acquis a priori, d'autre part, «tout phénomène de genre [étant] bien évidemment /... / en lui-même fondamentalement dépendant d'une culture» (Bouquet 1998, p. 119). Il s'agit donc d'isoler, dans un premier temps et pour constituer le corpus, des catégories dont seule la description ultérieure peut prouver ou non le bien-fondé. Autrement dit, on se trouve face au dilemme de devoir disposer déjà d'un corpus représentatif pour pouvoir prendre une décision quant aux critères de classification devant mener justement à l'établissement du corpus. Pour construire néanmoins une conception opéra-

\footnotetext{
${ }^{1}$ Le «niveau de représentativité » est le niveau de fonctionnement langagier qu'on cherche à décrire dans une recherche donnée, autrement dit le niveau auquel on rapporte comme étant représentatifs les phénomènes langagiers que la recherche a mis au jour (cf. von Münchow 2004a, pp. 22-23).

${ }^{2}$ Rastier (2004, p. 120) distingue les genres des «champs génériques (ex. théâtre, poésie, genres narratifs)» et des « discours (ex. juridique $v s$ littéraire $v s$ scientifique) ».
} 
toire du genre en linguistique de discours comparative, je me suis servie des notions d'éticité et d'émicité, empruntées à Pike $(1967)^{3}$ : en choisissant des documents relevant de ce qu'on pense être un même genre, l'on aborde le genre dans une perspective étique ; l'analyse met alors à la disposition du chercheur les critères linguistiques et extralinguistiques pour définir le genre émique, concept qui permet de rejuger l'attribution étique du caractère générique à tel ensemble de documents, voire de reconsidérer la pertinence de l'analyse elle-même.

Pour décider de l'existence d'un genre étique et de l'appartenance de tel document à ce même genre, on peut avoir recours aux désignations ordinaires, mais j'utilise également le critère de la macrofonction supposée, ainsi que les cinq paramètres que propose Maingueneau (1996, p. 44) pour définir un genre, à savoir le statut respectif des locuteurs et des récepteurs ${ }^{4}$, les circonstances temporelles et locales de l'énonciation, le support et les modes de diffusion, les thèmes qui peuvent être introduits et, enfin, la longueur et le mode d'organisation.

En ce qui concerne les « jugements de non-émicité » après analyse, on les réserve pour les cas où l'écart entre tel exemplaire ayant été inclus dans le corpus comme appartenant au "genre étique » et les autres exemplaires du même corpus s'avère extrêmement important ${ }^{5}$ ou encore pour les cas où les manifestations d'un même genre étique dans deux communautés ethnolinguistiques se distinguent fondamentalement. En dehors de ces cas extrêmes, il semble plus raisonnable, plutôt que de prendre une quelconque "décision d'émicité », de délimiter différentes zones : les caractéristiques translangagières du corpus relèvent alors du «noyau émique » du genre sur le plan translangagier alors que les traits qu'ont en commun la grande majorité des exemplaires d'un genre à l'intérieur d'une communauté ethnolinguistique constituent le « noyau émique » du genre sur un plan « unilangagier».

En aval de l'analyse, le problème méthodologique majeur qui se pose est la question de savoir lesquels parmi les résultats de l'analyse sont à considérer comme caractéristiques du niveau de représentativité visé, à savoir un genre donné, et lesquels se rapportent à d'autres niveaux de représentativité (langue, discours, production individuelle, etc.). Alors qu'un corpus représentatif doit permettre de cerner les niveaux de représentativité « au-dessous » du genre, comme un sous-genre (la brève ou le reportage, par exemple, à l'intérieur du genre « journal télévisé »), un sous-ensemble de documents (par exemple, les journaux télévisés des chaînes privées par opposition à ceux des chaînes publiques) ou encore une production individuelle, ce n'est qu'au terme d'un grand nombre d'analyses de genres fort divers, appartenant à différents «champs» et «discours», au sens de Rastier, qu'on pourra émettre un jugement un

\footnotetext{
${ }^{3}$ Pike utilise l'opposition etic/emic (comme dans phonetic/phonemic, en anglais), qu'on peut traduire par étique/émique, pour distinguer des classifications généralisées "brutes» (etic) d'analyses systémiques plus spécifiques (emic) ou encore une perspective fondée sur des catégories extrinsèques au système à décrire (etic) à une perspective fondée sur des catégories intrinsèques (emic).

${ }^{4}$ Maingueneau utilise les termes énonciateurs et coénonciateurs.

${ }^{5}$ Cela a été le cas du journal télévisé Soir 3, appartenant au «genre étique » journal télévisé, mais que j'ai éliminé du «genre émique » après l'analyse du corpus de journaux télévisés français et allemands (cf. von Münchow 2001, 2004a) parce que le nombre de différences par rapport aux autres exemplaires de journaux télévisés était très élevé.
} 
tant soit peu informé sur l'attribution de tel ou tel phénomène langagier à tel genre ou alors à un discours ou une langue-culture en général, voire à un ensemble de languescultures. En ce sens, ce qui est exposé infra est à comprendre comme se situant sur le chemin entre «l'étique » et «l'émique » : le cumul des descriptions/interprétations de différents genres discursifs me permet d'établir des hypothèses argumentées concernant le niveau de représentativité auquel j'attribue tels résultats d'analyse, hypothèses dont on ne peut cependant exclure la mise en cause par des analyses ultérieures.

Ainsi, en linguistique de discours comparative, «le genre » en tant que concept est avant tout une catégorie opératoire et non l'objet de recherche, pas plus que ne l'est la description/interprétation des caractéristiques de tel ou tel genre, en fin de compte. Ces dernières constituent cependant un objet de recherche intermédiaire sans lequel l'objet à long terme, la description/interprétation de différentes cultures discursives relevant de communautés ethnolinguistiques diverses ne peut être atteint. Je mets donc en œuvre une méthodologie «bottom-up », si l'on veut, la description/interprétation d'une série de genres discursifs - série limitée car il s'agit d'une approche qualitative devant mener à des connaissances sur différentes cultures discursives.

\subsection{Corpus analysés et catégories descriptives}

Les phénomènes langagiers qui sont exposés infra font partie des résultats de l'analyse comparative des cinq corpus suivants, en trois langues: 1) 30 journaux télévisés français et allemands datant de 1993 ; 2) environ 6000 messages en français et en anglais postés sur des forums de discussion sur internet se rapportant au thème de l'environnement ; 3) six manuels scolaires de français et d'allemand langue maternelle pour le niveau Cours Élémentaire 1, publiés entre 1994 et 2000 ; 4) onze manuels scolaires de français et d'allemand langue maternelle pour le niveau Troisième, publiés entre 1998 et 2003 ; 5) six livres de puériculture français et allemands, publiés entre 1973 et 2004, les éditions analysées se situant entre 2000 et 2004. Ces corpus se rapportent tous à des genres de l'écrit ou de ce qu'on peut appeler "écrit à oraliser ", dans le cas du journal télévisé.

Bakhtine définit le genre comme « un type d'énoncé donné, relativement stable du point de vue thématique, compositionnel et stylistique» (1984, p. 269). Les « unités compositionnelles » sont définies ainsi (ibid.) : " type de structuration et de fini d'un tout, type de rapport entre le locuteur et les autres partenaires de l'échange verbal (rapport à l'auditeur, ou au lecteur, à l'interlocuteur, au discours d'autrui, etc.). » Dans cet article, ce sont les «types de structuration d'un tout» et les «types de rapport au discours d'autrui » dont il sera question de façon centrale. Plus précisément, les catégories descriptives à l'aide desquelles on comparera les manifestations des différents genres mentionnés sont les types de séquences textuelles et le discours rapporté.

Pour l'étude des types séquentiels, je me suis inspirée des travaux d'Adam (notamment 1992), tout en apportant des modifications substantielles à ses propositions. Les types séquentiels, qui sont de nature prototypique, se définissent par l'organisation de leurs énoncés (ou macro-propositions) entre eux, un texte consistant généralement en plusieurs séquences de types différents. On fera ici état des types narratif, descriptif, argumentatif, explicatif, procédural, prédictif et métatextuel (cf. von Münchow 2004a, pp. 73-91). 
S'agissant du discours rapporté, qui constitue la deuxième catégorie descriptive retenue, je le définis, en me référant aux travaux d'Authier-Revuz (notamment 2001), comme « opération métadiscursive de représentation d'un acte d'énonciation par un autre acte d'énonciation ». Parmi les types de base qui constituent le champ (cf. AuthierRevuz, op. cit., pp. 194-195), il sera ici essentiellement question des discours direct, indirect et bivocal. Combiner l'étude des types séquentiels et celle du discours rapporté permet d'exploiter, pour reprendre la terminologie de Courtine (1989, p. 12), l'axe « horizontal » (c'est-à-dire le « déroulement » intratextuel) aussi bien que l'axe « vertical » (à savoir les relations intertextuelles) d'un document, ce qui parait constituer une approche d'ensemble faisant preuve d'une certaine complétude.

En me fondant sur les cinq genres étudiés, je mettrai en évidence quelques-uns des traits constituant les «stabilités » relevées à différents niveaux de représentativité, chacun des niveaux participant à la mise au jour des caractéristiques spécifiquement génériques, que ce soit sur un plan translangagier ou unilangagier, de même qu'à la construction d'un savoir naissant sur le niveau de représentativité visé in fine par la linguistique de discours comparative : celui d'une culture discursive relevant d'une communauté ethnolinguistique. À chaque niveau, je proposerai des hypothèses interprétatives permettant d'expliquer les stabilités, interprétations qui seront reliées à des causalités linguistiques, techniques, institutionnelles, culturelles, etc. ${ }^{6}$

\section{Stabilités translangagières}

«La diversité fonctionnelle, se dira-t-on, fait que les traits communs à tous les genres du discours sont abstraits et inopérants », avance Bakhtine (1984, p. 266). En effet, la partie essentielle des « stabilités » observées lors de l'analyse des corpus que j'ai récoltés se situe au niveau générique translangagier ou unilangagier. On constate néanmoins, s'agissant de la distribution des différents types séquentiels dans les genres que j'ai analysés, l'omniprésence de la séquence descriptive. À moins de chercher la raison pour cet état de fait dans l'analyse même - la «faible caractérisation séquentielle » du type descriptif (Adam 2005, p. 146) pouvant favoriser un classement par défaut de tel élément textuel comme étant descriptif - on peut supposer que notamment le « décrire perceptuel», que Vogeleer (1992) oppose au "décrire épistémique», s'oppose à un type de séquence comme l'explication en ce qu'il n'implique pas l'instauration d'une hiérarchie cognitive entre locuteur et destinataire. En effet, tout en ayant un certain pouvoir, en tant que «maitre de son plan» (Adam 1992, p. 77), celui qui décrit ne sait pas forcément plus que celui à qui est adressée la description : il voit (et/ou il entend) et il montre. Tout en supposant un "point de vue », la description est par ailleurs plus neutre que l'argumentation car elle ne sollicite pas de jugement de la part du locuteur ni du destinataire.

\footnotetext{
${ }^{6}$ La partie interprétative ne sera qu'esquissée ici, le cadre de cet article ne permettant d'exposer que très peu de résultats d'analyse. En effet, les hypothèses sur des causalités techniques, linguistiques, culturelles, etc. doivent être reliées, pour être étayées, à un réseau de propriétés et non se rattacher à une propriété unique, mise en réseau que j'effectue dans des monographies se rapportant à chacun des genres.
} 


\section{Stabilités unilangagières transgénériques}

Selon Bakhtine (1984, pp. 284-285), les genres sont «parfois standardisés et stéréotypés, parfois plus souples, plus plastiques et plus créatifs. » La " prescriptivité » est un critère qui permet, certes, d'opposer différents genres discursifs, mais une différence dans le «degré de prescriptivité» marque déjà le rapport entre les genres allemands et les genres français dans leur ensemble. En effet, tous les genres analysés se sont avérés plus homogènes en France qu'en Allemagne, constituant ainsi des catégories plus contraignantes. Autrement dit, le contexte générique se fait davantage ressentir dans un document individuel français que dans un texte allemand.

Un deuxième type de stabilités transgénériques est à relier à des contraintes linguistiques à proprement parler. On constate en effet dans le corpus allemand une plus grande présence du discours indirect que dans le corpus français, distribution qu'on peut mettre en rapport avec la possibilité de clairement marquer le discours indirect à l'aide d'un subjonctif seulement - et donc sans introducteur ou modalisateur, ce qui constitue un facteur d'économie syntaxique considérable. Comme le constate déjà Guenther (1927, p. 70), cette possibilité semble mener à une utilisation plus étendue du discours indirect en allemand que dans les langues romanes.

\section{Stabilités génériques translangagières}

À l'opposé des deux niveaux abordés supra, on relève au niveau générique d'innombrables «stabilités », aussi bien sur le plan séquentiel que sur celui du discours rapporté, dont on exposera ici quelques-unes.

\subsection{Degré de prescriptivité}

Les différents genres peuvent être situés sur l'échelle suivante, allant d'une ritualisation maximale vers une prescriptivité minimale :

- manuel scolaire pour le niveau Cours Élémentaire 1,

- manuel scolaire pour le niveau Troisième,

- journal télévisé,

- livre de puériculture,

- forum de discussion.

En dehors du genre «forum de discussion», qu'on peut considérer comme «conversationnel» (Maingueneau 2004, p. 110) et qui n'exerce que des contraintes (relativement) faibles sur la construction des documents y appartenant, on a affaire exclusivement à des genres que Maingueneau (op. cit., p. 112) classerait parmi les « genres institués de mode (2)». Parmi les différentes catégories qu'il établit, "selon la relation qui s'établit entre ce [qu'il] appel[le] 'scène générique' et 'scénographie'» (op. cit., p. 111), autrement dit en se fondant essentiellement sur le critère de la ritualisation plus ou moins grande, les « genres institués de mode (2)» «suivent en général une scénographie préférentielle, attendue, mais ils tolèrent des écarts, c'est-à-dire le recours à des scénographies plus originales » (op. cit., p. 112). Les contraintes exercées par l'institution scolaire rendent néanmoins les manuels mentionnés bien plus homogènes que les journaux télévisés, ces derniers formant un ensemble moins hétérogène que les livres de puériculture, pour lesquels la notion d'« auteur » commence à prendre un véritable sens. 


\subsection{Distribution des types séquentiels et des occurrences de discours rapporté}

\subsubsection{Distribution des types séquentiels}

La répartition des différents types séquentiels à l'intérieur d'un même genre est relativement stable, aussi bien sur le plan d'une seule langue-culture qu'au niveau translangagier :

- le journal télévisé comporte une majorité de séquences descriptives, mais aussi une part importante de séquences narratives et/ou argumentatives ; l'explication est plus discrète, mais néanmoins présente, ainsi que le type prédictif ; enfin, on relève un nombre important de séquences métatextuelles ${ }^{7}$;

- dans le manuel scolaire, les éléments textuels autres que les textes « extérieurs » donnés à étudier et qui sont suffisamment longs pour pouvoir développer une séquentialité à proprement parler sont notamment descriptifs ou procéduraux; on relève également quelques narrations, l'argumentation étant rare, mais présente ;

- dans les livres de puériculture, la description est accompagnée d'argumentations, d'explications et de séquences procédurales, les narrations étant rares ;

- dans les forums de discussion, l'argumentation est majoritaire, la description étant également très présente, alors que la narration est plus rare et que l'explication se fait discrète ; le type procédural peut également être relevé.

Cette énumération confirme la nécessité, indiquée par Beacco (2004, p. 113), de " distinguer la consistance d'une régularité (sa masse linguistique quantifiable) et sa représentativité (son caractère de marqueur générique), puisque la présence importante d'un signe ou d'un ensemble de signes n'est pas nécessairement un trait distinctif générique. » Et c'est justement la comparaison des différents genres qui permet d'affirmer que malgré ou plutôt à cause de sa présence importante dans tous les genres, la séquentialité descriptive ne caractérise aucun genre. En revanche, un type présent en quantité faible, mais dont l'apparition est régulière dans les documents relevant de tel genre, peut être caractéristique de ce dernier, comme l'est la séquence métatextuelle dans le cas du journal télévisé. La régularité d'apparition de cette séquence souligne le fait qu'il ne s'agit pas seulement, dans le journal télévisé, d'informer le public, mais aussi, ou peut-être surtout, de mettre en valeur le travail journalistique accompli.

Il est également important de vérifier quels types de séquences constituent des séquences principales (ou « enchâssantes »), des subordonnées (ou " enchâssées ») ou des dominantes ${ }^{8}$. Les types fournissant de façon récurrente des dominantes dans un genre donné peuvent être considérés comme valorisants pour ce genre alors que la distribution préférentielle d'un type en subordonnée peut constituer un indice de sa non-légitimité.

\footnotetext{
${ }^{7}$ Il s'agit de séquences qui entrent en rapport avec le document dans son ensemble et qui contiennent des informations sur sa construction (journalistique, en l'occurrence). Voir von Münchow 2004a, pp. 80-81 pour une définition plus complète.

${ }^{8}$ Dans l'analyse, je m'efforce de relever dans chacun des documents constituant le corpus une (ou deux) «dominante(s) séquentielle(s) », à l'aide de la combinaison de trois critères: le(s) type(s) de séquence(s) majoritaire(s) parmi les séquences principales; le(s) type(s) occupant le plus d'espace textuel, principales et subordonnées confondues; le(s) type(s) occupant les "positions stratégiques » du document, à savoir le début et la fin (cf. von Münchow 2004a, p. 81).
} 
Dans le cadre du journal télévisé, par exemple, la narration fournit régulièrement des principales aussi bien que des subordonnées, mais rarement des dominantes, ce qui montre que son apparition est nécessaire et légitime, certes, mais non particulièrement valorisante. L'argumentation, en revanche, apparait relativement souvent en dominante, sans pour autant être plus récurrente que la narration sur le plan quantitatif, ce qui indique que sa mise en évidence semble profiter à l'attrait du journal télévisé. On peut en effet supposer que l'argumentation propose au spectateur l'image valorisante d'une personne critique par rapport au monde, à égalité et en interaction avec le journaliste alors que la narration assigne peut-être une image trop passive au spectateur. S'agissant enfin de l'explication, elle semble indispensable dans le journal télévisé, vu la régularité de sa présence, mais ne peut le plus souvent se loger (ou «se cacher») que dans des subordonnées, tant est désobligeante (du moins potentiellement) l'image d'un spectateur infériorisé par un journaliste qui connaît une certaine causalité et qui fait état de ce savoir.

\subsubsection{Distribution $d u$ discours rapporté}

Le discours rapporté est présent dans tous les genres analysés, sa distribution étant la plus réduite dans le forum de discussion et la plus importante dans le journal télévisé et dans le livre de puériculture, qui en sont saturés. Ce qui caractérise surtout les différents genres du point de vue du discours rapporté, c'est le degré de marquage de ce dernier, ainsi que les « locuteurs rapportés ». Dans le journal télévisé, le discours rapporté est le plus souvent clairement marqué comme tel et les «locuteurs rapportés », à savoir majoritairement les acteurs de la politique et de l'économie, sont identifiés de façon univoque. Dans les livres de puériculture, en revanche, un discours rapporté clairement marqué dont les «locuteurs rapportés » sont les spécialistes (médecins, psychologues, éducateurs, etc.) de l'enfance côtoie de nombreuses occurrences de discours rapporté non ou peu marquées dont on a tendance à attribuer la prise en charge énonciative aux enfants, aux parents, à l'entourage des parents, etc. Dans les manuels scolaires, on rapporte, de façon clairement marquée, surtout la parole des auteurs dont les textes sont reproduits, mais aussi, de façon très récurrente, celle de l'élève : il s'agit alors généralement d'un discours rapporté sous forme d'injonction ou d'interrogation par lequel on demande à l'élève de produire du discours.

Les proportions des différents types de discours rapporté, quant à elles, varient considérablement d'un document à l'autre, de même que d'une communauté ethnolinguistique à l'autre. Le seul type de base dont la distribution permet de caractériser les différents genres est celui qu'on appelle généralement discours indirect libre, mais qui parait mieux nommé par la désignation discours bivocal ${ }^{9}$. Le discours bivocal relève, « [c]ontrairement à l'unité du discours indirect et à la bipartition du discours direct /.../ d'un 'parler ensemble' pour les deux voix, rapporteur et rapporté, qui, sans la hiérarchisation (base du rapporteur avec interférences) de la modalisation, apparaît comme un mode original de représentation d'un discours autre » (Authier-Revuz 2001, p. 201). À l'opposé des autres types, le discours bivocal se présente obligatoirement sans introducteur ni modalisateur et aucune séquence «énoncé rapporté » n’est

\footnotetext{
${ }^{9}$ Bakhtine (1978, p. 144) réserve cette appellation au roman, mais on peut également l'utiliser pour d'autres genres.
} 
délimitée (voire délimitable) à l'intérieur d'un énoncé englobant ${ }^{10}$. Ce «type à part» de discours rapporté est très rare dans les genres que j'ai étudiés, voire absent, sauf dans le livre de puériculture, où il est extrêmement récurrent :

Exemple 1.

Le chat risque-t-il d'étouffer votre bébé dans son berceau ?

[Antier, E. (1999), Élever mon enfant aujourd'bui, Paris, Robert Laffont, éd. 2003, p. 17]

Pourquoi le nom «chat » est-il déterminé par l'article défini dans cet intertitre, alors qu'aucun chat n'a été précédemment évoqué ? Ce déterminant ne peut être considéré que comme se rapportant à une situation d'énonciation dans laquelle on n'a pas besoin de spécifier de quel chat il s'agit, étant donné qu'il n'y en a qu'un : c'est « le chat de la maison » appartenant à la lectrice, cette dernière étant responsable de la détermination, alors que le déictique "votre » est clairement assumé par l'auteure locuteur rapportant. La lectrice-mère étant également responsable de la prise en charge de la modalité de phrase interrogative, on a affaire à un mélange de voix qui véhicule l'impression que l'auteure partage l'intimité de sa lectrice. La création de cette relation de proximité semble être la principale finalité de l'emploi du discours bivocal dans le livre de puériculture.

\section{Stabilités génériques unilangagières}

La comparaison des manifestations d'un même genre discursif aboutit aussi à la mise au jour d'un grand nombre de différences entre les communautés ethnolinguistiques, différences constituant à leur tour des "stabilités génériques unilangagières ». Je n'aborderai ici que la question du degré de marquage des énoncés comme relevant du discours rapporté dans les forums de discussion français et anglo-américains.

Dans le forum français fr.soc.environnement, le discours rapporté se présente majoritairement sous forme d'allusions, dont certaines ne sont compréhensibles que pour les usagers du forum. Le message suivant s'inscrit dans une discussion qui s'est déplacée du problème du tabagisme passif vers la question de la légitimité des interdictions dans la société. Le locuteur répond à un autre locuteur en se référant ironiquement à la position précédemment exposée d'un troisième locuteur qui défendait les vertus de l'interdiction ${ }^{11}$ :

\footnotetext{
10 Pour une présentation plus détaillée du phénomène, voir von Münchow 2001, pp. 444-446, 488491.

${ }^{11}$ L'orthographe des messages est reproduite telle quelle.
} 
Exemple 2. Extrait du fil de discussion «Tabagisme passif et droit des non fumeurs » (12/6/01 11:13). De : A

$$
/ \ldots /
$$

(i) Le monsieur, il t'explique que l'humanité, ça se caractérise par l'interdiction, il l'a lu chez les anthropologues, (ii) faut vraiment etre un post-soixante-huitard drogué, chevelu et irresponsable pour aller contre cet argument d'autorité. L'interdiction devrait etre la norme, on ne le répètera jamais assez, de meme que la liberté, c'est l'esclavage. / .../

Après un discours indirect (i), on relève ici un discours direct libre (ii) - c'est-àdire un discours direct sans introducteur - dont les locuteurs rapportés seraient les personnes défendant l'interdiction de fumer dans les lieux publics et plus généralement la nécessité de certaines interdictions dans la société. Les participants du forum reconnaitront d'ailleurs l'énoncé «L'interdiction devrait être la norme » comme ayant effectivement été posté antérieurement dans le même fil de discussion. En effet, les nombreuses allusions du corpus relèvent généralement du discours rapporté «intraforum » et sont le plus souvent puisées dans l'interdiscours que constituent divers discours "de gauche ", écologistes et notamment "soixante-huitards ", tantôt revendiqués, tantôt rejetés.

À l'opposé du forum francophone, l'allusion n'est pas appréciée dans le forum anglophone talk.environment; le discours rapporté est le plus souvent clairement marqué. Par l'intermédiaire de discours indirects reformulant la parole des autres ou, très souvent, du locuteur même, les participants essaient de (faire) comprendre les différentes opinions énoncées :

Exemple 3. Extrait du fil de discussion «Farmers Are Getting Angry over GE Crops » (16/4/01 14h35). De : B

$$
/ \ldots /
$$

>>>>>> [B parle] /.../ It was (i) C's remark that 'folks live longer' on GE human insulin. that struck me as odd. /.../

>>>>> [C parle $] / . . . /$ (ii) I should have said since human insulin result in better control of blood sugar less adverse events are encountered. /.../

$>>$ B parle $] / \ldots /$ (iii) the poster your are referring to spelled out for you rather clearly, that he is not using human insulin. ((iv) he wrote that he is using a Humalog/Ultralente combination, both of which preparations have been modified to have other properties than human insulin). / .../ 
$[/ \ldots /$

>>>>>> [B parle] /.../ C'était (i) la remarque de $\mathrm{C}$ que 'les gars vivent plus longtemps' avec de l'insuline humaine génétiquement modifiée que j'ai trouvée bizarre. /.../

>>>>> [C parle] /.../ (ii) J'aurais dû dire puisque l'insuline humaine entraine un meilleur contrôle du sucre sanguin il arrive moins d'accidents malencontreux. /.../

$>>[\mathrm{B}$ parle $] / / \ldots / /$ (iii) le participant auquel tu te réfères te disait assez clairement qu'il n'utilise pas d'insuline humaine. ((iv) il disait qu'il utilisait une combinaison d'Humalog et d'Ultralente, les deux préparations ayant été modifiées pour présenter des propriétés autres que celles de l'insuline humaine). / .../]

Dans cet échange entre B et C, le discours indirect (i), comportant un îlot textuel marqué par des guillemets simples, sert à rapporter un énoncé de $\mathrm{C}$ dont $\mathrm{B}$ met en question la validité. C réagit en énonçant, sous forme d'un discours indirect dont il est lui-même le «locuteur rapporté » hypothétique (ii), une reformulation plus complète du point de vue explicatif de son énoncé qui a été critiqué. Un échange plus tard, B revient à la charge en faisant remarquer, par l'intermédiaire des discours indirects (iii) et (iv), que certains éléments du discours auquel se réfère $C$ sont en contradiction avec ce que ce dernier affirme.

Dans l'ensemble, le discours rapporté indirect sert donc ici essentiellement à clarifier le cours de la discussion. À travers la compréhension des énoncés se construit celle des faits présentés, souvent très complexes sur talk.environment, forum dont l'objectif semble être essentiellement de nature cognitive alors que les locuteurs du forum français donnent l'impression de poursuivre un but communicatif, à savoir le fait de renforcer les liens entre les membres de leur communauté électronique « de connivence » à l'aide d'allusions ne pouvant être comprises que par eux.

\section{Stabilités de sous-ensembles et de productions individuelles}

Les résultats de l'analyse des types séquentiels et du discours rapporté dans un genre permettent souvent d'opposer certains exemplaires du genre à d'autres, au niveau unilangagier ou translangagier. La nature des ensembles ainsi formés donne de précieux renseignements sur le genre en question et permet d'établir des hypothèses interprétatives qu'on peut souvent relier à des causalités de différents types.

On constate ainsi une différence importante entre les journaux télévisés de chaînes publiques et de chaînes privées en Allemagne, alors qu'il ne pourrait être question que d'une différence légère en France. Les chainnes publiques et les chaînes privées constituent donc des configurations bien distinctes dans les deux pays, fait qu'on peut relier à la façon dont a été créé le secteur privé de part et d’autre du Rhin : en France, 
c'est la privatisation d'une chaîne publique qui a inauguré le processus de «dérégulation » alors que cette dérégulation s'est manifestée à travers la création progressive de nouvelles chaînes privées en Allemagne (cf. von Münchow 2004a, pp. 187-191).

Parmi les livres de puériculture, les ouvrages allemands d'auteurs hommes s'opposent clairement à celui qui est écrit par une femme alors qu'on ne peut pas délimiter de sous-ensembles correspondants dans le corpus français. Les auteurs hommes allemands tiennent en effet de façon récurrente des propos à orientation « éthique ». L'un des procédés s'inscrivant dans cette orientation est l'argumentation fondée sur des prédictions concernant l'avenir de l'enfant, autrement dit l'emploi d'une séquence argumentative qui régit une subordonnée prédictive. C'est le cas dans l'extrait suivant, dont la conclusion argumentative (vii) est reconnaissable grâce à la modalité déontique (en italiques) :

Exemple 4.

(i) Ihr Kind empfindet seinen Körper als feindlich, (ii) wenn er ihm Unruhe, Unlustgefühle oder Schmerzen bereitet. Das ist der Fall, wenn es Hunger hat, schwitzt oder friert usw. (iii) Wenn Sie dann nicht zu Hilfe kommen, (iv) verfestigt sich diese Unzufriedenheit mit dem eigenen Körper. (v) Erlebt ein Kind häufig solche Enttäuschungen, (vi) dann kann es auch keine freundliche Einstellung zur Außenwelt (die ihm ja nicht hilft) entwickeln, es wird sich resigniert abwenden. (vii) Deshalb sollten Sie Ihr Kind besonders im ersten Lebensjahr, solange es noch nicht warten kann (also solange es noch nicht sicher ist, dass schon irgendjemand bald kommt), möglichst viele Erfahrungen machen lassen, die sein Vertrauen in die Welt wachsen lassen und ihm Erlebnisse ersparen, die zu Misstrauen führen. [Diekmeyer, U. (1973), Das Elternbuch 1. Unser Kind im ersten Lebensjahr, Reinbek, Rororo, éd. 2002, p. 251]

[(i) Votre enfant ressent son corps comme lui étant hostile (ii) lorsqu'il lui procure de l'agitation, des sentiments d'ennui ou des douleurs. C'est le cas lorsqu'il a faim, lorsqu'il transpire ou qu'il a froid, etc. (iii) Si vous ne lui venez pas en aide à ces moments-là, (iv) l'insatisfaction face à son propre corps se renforce. (v) Si un enfant vit souvent ce genre de déceptions, (vi) il ne peut pas développer d'attitude amicale envers son entourage (puisque ce dernier ne l'aide pas), il se détournera avec résignation. (vii) C'est pour cela que vous devrię faire en sorte que votre enfant ait, notamment pendant sa première année, tant qu'il est encore incapable d'attendre (c'est-à-dire tant qu'il ne sait pas avec certitude que quelqu'un arrivera sûrement bientôt), autant d'expériences que possible qui fassent croître sa confiance en le monde et qui lui évitent des événements qui mènent à la méfiance.]

Une première conséquence (i) précède la condition (ii) et entraîne une nouvelle condition (iii) déclenchant à son tour une conséquence (iv), à partir de laquelle est construite une troisième condition (v), de nouveau suivie d'une conséquence (vi). 
L'ensemble de la séquence prédictive sert alors d'argument pour la conclusion (vii). Pour résumer, l'auteur essaie de convaincre la mère, qui est le destinataire principal de l'ouvrage, de réparer très vite tous les désagréments auxquels son enfant de moins d'un an est soumis en lui prédisant que si elle ne le fait pas, son enfant ne pourra pas développer d'attitude positive envers son entourage et se réfugiera dans la résignation. Dans l'ensemble, ce type de séquences prédictives, fort récurrentes et dont la plupart correspondent à des scénarios-catastrophe, crée de l'inquiétude chez la mère, de même qu'un sentiment de culpabilité. Les auteurs n'essaient pas de rassurer la mère, comme c'est le cas dans tous les autres livres de puériculture, mais de la convaincre de «bien » faire en lui faisant peur.

Cette tendance forte dans les livres d'auteurs hommes peut expliquer une particularité récurrente de l'ouvrage allemand écrit par une femme ${ }^{12}$ : l'argumentation prouvant l'inutilité, voire le caractère nocif d'un sentiment de culpabilité. Ainsi la description, dans l'extrait suivant, d'une situation d'énervement (i), description comportant un exemple tiré de l'expérience personnelle de l'auteure, est suivie d'un argument (ii), sans conclusion, dans lequel elle avance l'affirmation qu'un sentiment de culpabilité ne ferait qu'aggraver encore l'état des choses :

Exemple 5.

(i) Nichts bringt uns so an die Grenzen unserer Nervenkraft wie ein schreiendes Kind, und in den ersten Lebensmonaten passiert das ziemlich häufig. Ich selber habe dabei selten herausgehört, was meinem Kind wirklich fehlte und konnte es oft nicht beruhigen. Gerade beim ersten Kind verfolgte mich sein Geschrei bis in meine Träume.

(ii) Doch ein schlechtes Gewissen, Gefühle des Versagens und Selbstvorwürfe belasten in dieser anstrengenden Zeit nur zusätzlich. [von Cramm, D. et Schmidt, E. (1995), Unser Baby. Das erste Jabr, Munich, Gräfe und Unzer, 12e éd. 2003, p. 72]

[(i) Rien ne nous pousse autant au bord de la crise de nerfs qu'un enfant qui crie et cela arrive assez souvent pendant les premiers mois de la vie. Moi-même, je n'ai que rarement été capable de comprendre ce que mon enfant avait vraiment et souvent je n'arrivais pas à le calmer. Surtout les cris du premier enfant me poursuivaient jusque dans mes rêves.

(ii) Mais une mauvaise conscience, des sentiments d'échec et des reproches adressés à soi-même ne font que constituer un poids supplémentaire pendant cette période épuisante.]

Cette particularité se situant au niveau des «stabilités de production individuelle » transcende en même temps ce niveau puisqu'elle s'inscrit, semble-t-il, dans un champ interdiscursif apparemment dominé par un discours créateur de culpabilité, discours contre lequel semble s'ériger l'auteure. Ainsi des caractéristiques «individuelles » sont

12 Le co-auteur masculin disparaît du cadre énonciatif dès les premières pages. 
tout à fait significatives pour l'analyse d'un genre en ce qu'elles permettent de mettre en évidence des tensions entre des positionnements majoritaires et minoritaires, voire de montrer une possible évolution du genre. Qui plus est, ce qui peut d'abord paraitre une caractéristique individuelle indique parfois la nécessité d'élargir le corpus, élargissement permettant éventuellement de découvrir que le trait en question constitue en réalité une caractéristique s'inscrivant dans un sous-ensemble n'apparaissant pas dans le premier corpus, de taille trop réduite.

\section{7. À la croisée des genres : soupçons de cultures discursives}

Pour terminer, je m'efforcerai de donner une idée de l'objet à long terme de la linguistique de discours comparative. Il s'agit ici de montrer, sous forme d'esquisse hypothétique, comment on peut dépasser la simple juxtaposition des résultats de l'analyse des différents genres et aller vers une mise en réseau des descriptions et interprétations proposées.

C'est par l'intermédiaire de la séquence explicative dans les genres français que j'aborderai brièvement ce sujet. Comme je l'ai déjà indiqué, l'emploi de la séquence explicative peut être délicat, sans doute à cause de la hiérarchie cognitive qu'elle instaure entre locuteur et récepteur, le premier apportant un savoir de type causal au deuxième. Ce problème ne se pose pas dans les livres de puériculture, dans lesquels la séquence explicative est très récurrente, en particulier dans le corpus français ${ }^{13}$. En ce qui concerne les journaux télévisés, la séquence explicative est plus récurrente dans le corpus français que dans le corpus allemand, tout en restant minoritaire dans l'ensemble. Enfin, dans les forums de discussion français, son emploi est sanctionné de façon négative par les autres participants quasi systématiquement alors que les locuteurs du forum anglophone l'utilisent plus souvent et sans se le voir reprocher. L'hypothèse interprétative qu'on peut en tirer porte sur le rapport entre hiérarchie sociale et hiérarchie discursive dans les genres français : dans des genres dont les participants ont des statuts hiérarchiques différents, comme les journaux télévisés, cette hiérarchie peut (ou doit) se manifester aussi dans le discours, du moins discrètement ; en revanche, dans les genres dont les participants ont un statut égal, comme le forum de discussion, aucun locuteur n'est censé «se hisser» au-dessus des autres par des moyens discursifs comme la séquence explicative. La comparaison aux autres communautés ethnolinguistiques n'est pas aisée, étant donné que toutes les recherches n'ont pas porté sur les mêmes communautés, mais le rapport entre hiérarchie extra-discursive et hiérarchie discursive semble moins étroit dans les communautés allemande et anglo-américaine ${ }^{14}$, une situation institutionnellement hiérarchique n'entraînant pas nécessairement un « discours à

\footnotetext{
13 Les manuels scolaires contiennent beaucoup de séquences procédurales, mais peu d'explication causale, ce qui est sans doute dû au fait que la thématique - il s'agit de manuels de lecture en langue maternelle - ne s'y prête pas.

${ }^{14}$ Certaines marques indiquent que les locuteurs du forum talk.environment sont majoritairement originaires des États-Unis, mais l'analyse d'un « genre électronique » pose bien entendu un problème d'identification des communautés ethnolinguistiques concernées.
} 
effet hiérarchique » et une situation égalitaire n'empêchant pas les différents locuteurs d'assumer momentanément une position cognitive plus élevée que les autres, par exemple en énonçant une séquence explicative.

Dans l'ensemble, je me suis efforcée de mettre en évidence le statut de tertium comparationis et de niveau de représentativité qu'a le genre discursif en linguistique de discours comparative ainsi que la valeur heuristique de la démarche comparative. J'ai montré que les «stabilités » qu'on peut constater sur le plan des types séquentiels et sur celui du discours rapporté dans cinq genres de l'écrit et dans trois communautés ethnolinguistiques se distribuent sur différents niveaux et contribuent ainsi à la description de chaque genre et à l'établissement d'hypothèses interprétatives concernant les fonctions du genre et les représentations (mentales) qui circulent parmi les locuteurs et les récepteurs, ainsi que les causalités institutionnelles, culturelles, etc. à mettre en relation avec les fonctions et les représentations en question.

\section{RÉFÉRENCES BIBLIOGRAPHIQUES}

ADAM, J.-M. (1992), Les textes: types et prototypes. Récit, description, argumentation, explication et dialogue, Paris, Nathan.

AdAm, J.-M. (2005), La linguistique textuelle. Introduction à l'analyse textuelle des discours, Paris, Armand Colin.

Adam, J.-M. et Heidmann, U. (2004), «Des genres à la généricité. L'exemple des contes (Perrault et Grimm) », Langages, $\mathrm{n}^{\circ} 153$, pp. 62-72.

Authier-Revuz, J. (2001), «Le discours rapporté » in Tomassone, R. (éd.) Une langue : le français, Paris, Hachette Éducation, pp. 192-201.

BAKHTine, M. (1978), Esthétique et théorie du roman, Paris, Gallimard, éd. or. 1975.

BAKTHINe, M. (1984), Esthétique de la création verbale, Paris, Gallimard, éd. or. 1979.

BEACCO, J.-C. (2004), «Trois perspectives linguistiques sur la notion de genre discursif», Langages, $\mathrm{n}^{\circ} 153$, pp. 109-119.

Bouquet, S. (1998), «Linguistique textuelle, jeux de langage et sémantique du genre », Langages, $\mathrm{n}^{\circ} 129$, pp. 112-124.

BOUQUET, S. (2004), «Sémiotique grammaticale et sémantique des (genres de) jeux de langage : les 'pronoms personnels' clitiques en français », Langages, $\mathrm{n}^{\circ}$ 153, pp. 28-40.

BRANCA-RosofF, S. (1999), «Des innovations et des fonctionnements de langue rapportés à des genres », Langage et société, $\mathrm{n}^{\circ}$ 87, pp. 115-129.

Bronckart, J.-P. (1997), Activité langagière, textes et discours, Lausanne-Paris, Delachaux et Niestlé.

BRONCKART, J.-P. (2004), «Les genres de textes et leur contribution au développement psychologique », Langages, $\mathrm{n}^{\circ} 153$, pp. 98-108. 
Courtine, J.-J. (1989), Corps et discours: éléments d'bistoire des pratiques langagières et expressives, présentation du dossier de thèse d'État sur travaux, Université Paris 10-Nanterre.

Dolinine, C. (1999), « Le problème des genres du discours quarante-cinq ans après Bakhtine », Langage et société, $\mathrm{n}^{\circ} 87$, pp. 25-40.

Guenther, W. (1927), Probleme der Rededarstellung. Untersuchungen zur direkten, indirekten und serlebten « Rede im Deutschen, Französischen und Italienischen, thèse de doctorat, Université de Berne.

Kerbrat-Orecchioni, C. et Traverso, V. (2004), «Types d'interactions et genres de l'oral », Langages, $\mathrm{n}^{\circ} 153$, pp. 41-51.

Maingueneau, D. (1996), Les termes clés de l'analyse de discours, Paris, Seuil, coll. « Mémo».

Maingueneau, D. (2004), «Retour sur une catégorie : le genre », in Adam, J.-M., GRIZE, J.-B. et Ali Bouacha, M. (éd.) Texte et discours : catégories pour l'analyse, Dijon, EUD, pp. $107-$ 118.

MALRIEU, D. (2004), «Linguistique de corpus, genres textuels, temps et personnes », Langages, $\mathrm{n}^{\circ} 153$, pp. $73-85$.

MoIRAND, S. (2003), "Quelles catégories descriptives pour la mise au jour des genres du discours ? ", conférence à la journée scientifique "Les genres de l'oral » à l'université Lyon 2-Lumière et ENS LSH, [http://gric.lyon2.fr/Equipe1/actes/journees_genre.htm].

MoIrAND, S. (2004), «Le dialogisme, entre problématiques énonciatives et théories discursives», Cabiers de praxématique, $\mathrm{n}^{\circ} 43$, pp. 189-220.

Moirand, S. (2006), «Textes/discours et co(n)textes », entretien, Pratiques, n 129-130, pp. 43-49.

VON MÜNCHOw, P. (2001), Contribution à la construction d'une linguistique de discours comparative: entrées dans le genre journal télévisé français et allemand, thèse de doctorat, Université Paris 3Sorbonne nouvelle.

VON MÜNCHOw, P. (2004a), Les journaux télévisés français et allemands. Plaisir de voir ou devoir de s'informer, Paris, Presses Sorbonne Nouvelle.

VON MÜNCHOW, P. (2004b), «Réflexions sur une linguistique de discours comparative : le cas du journal télévisé », TRANEL, $\mathrm{n}^{\circ}$ 40, pp. 47-70.

VON MÜNCHOW, P. (2004c), «Comparaison de manuels de français et d'allemand langue maternelle : représentations de l'enfant, de l'adulte, de l'apprentissage et de la langue », Marges Linguistiques, [http://www.marges-linguistiques.com].

VON MÜNCHOw, P. (2005), «L'enfant, l'adulte, l’apprentissage et la langue dans des manuels de français et d'allemand langue maternelle ", in BEACCO, J.-C., CHISs, J.-L., Cicurel, F. et VéroniQue, D. (éd.) Les cultures éducatives dans l'enseignement des langues, Paris, P.U.F., pp. 137-158.

VON MÜNCHOW, P. (2007), «Les représentations du père dans les guides parentaux français et allemands », in Sellenet, C. (éd.) Les pères en débat. Regards croisés sur la condition paternelle en France et à l'étranger, Ramonville Saint-Agne, Érès, pp. 125-136. 
von MünChow, P. et Rakotonoelina, F. (2006a) (éd.) Les Carnets du Cediscor, n 9, «Discours, cultures, comparaisons », Presses Sorbonne Nouvelle.

VON MÜnChow, P. et Rakotoelina, F. (2006b), «L'interrogation et le discours rapporté dans les forums de discussion sur l'environnement en français et en anglo-américain ", Les Carnets du Cediscor, n 9, pp. 93-112.

PIKE, K.L. (1967), Language in Relation to a Unified Theory of the Structure of Human Behaviour, La Haye, Mouton.

RAstier, F. (1998), «Le problème épistémologique du contexte et le statut de l'interprétation dans les sciences du langage », Langages, n 129 , pp. 97-111.

Rastier, F. (2004), « Poétique et textualité », Langages, n 153, pp. 120-126.

VogeleER, S. (1992), «La relation point de vue et son application aux phrases existentielles initiales ", in de Mulder, W. (éd.) Énonciation et parti pris, Amsterdam, Rodopi, pp. 349355. 
